\title{
Dahlberg-Borer-Newcomer syndrome
}

\author{
INSERM
}

\section{Source}

INSERM. (1999). Orphanet: an online rare disease and orphan drug data base. DahlbergBorer-Newcomer syndrome. ORPHA:1563

Dahlberg-Borer-Newcomer syndrome is a very rare ectodermal dysplasia syndrome, described in 2 adult brothers, characterized by the association of hypoparathyroidism, nephropathy, congenital lymphedema, mitral valve prolapse and brachytelephalangy. Additional features include mild facial dysmorphism, hyperthricoses, and nail abnormalities. 